\title{
Sequence analyses of the distal-less homeobox gene family in East African cichlid fishes reveal signatures of positive selection
}

\author{
Eveline T Diepeveen ${ }^{*}$, Fabienne D Kim and Walter Salzburger
}

\begin{abstract}
Background: Gen(om)e duplication events are hypothesized as key mechanisms underlying the origin of phenotypic diversity and evolutionary innovation. The diverse and species-rich lineage of teleost fishes is a renowned example of this scenario, because of the fish-specific genome duplication. Gene families, generated by this and other gene duplication events, have been previously found to play a role in the evolution and development of innovations in cichlid fishes - a prime model system to study the genetic basis of rapid speciation, adaptation and evolutionary innovation. The distal-less homeobox genes are particularly interesting candidate genes for evolutionary novelties, such as the pharyngeal jaw apparatus and the anal fin egg-spots. Here we study the $d l x$ repertoire in 23 East African cichlid fishes to determine the rate of evolution and the signatures of selection pressure.
\end{abstract}

Results: Four intact $d / x$ clusters were retrieved from cichlid draft genomes. Phylogenetic analyses of these eight $d / x$ loci in ten teleost species, followed by an in-depth analysis of 23 East African cichlid species, show that there is disparity in the rates of evolution of the $d / x$ paralogs. D/x3a and $d / x 4 b$ are the fastest evolving $d / x$ genes, while $d / x 1 a$ and $d / x 6 a$ evolved more slowly. Subsequent analyses of the nonsynonymous-synonymous substitution rate ratios indicate that $d|x 3 b, d| x 4 a$ and $d / x 5 a$ evolved under purifying selection, while signs of positive selection were found for $d / x 1 a, d / \times 2 a$, $d \times 3 a$ and $d 1 \times 4 b$.

Conclusions: Our results indicate that the $d / x$ repertoire of teleost fishes and cichlid fishes in particular, is shaped by differential selection pressures and rates of evolution after gene duplication. Although the divergence of the $d / x$ paralogs are putative signs of new or altered functions, comparisons with available expression patterns indicate that the three $d \mid x$ loci under strong purifying selection, $d \mid x 3 b, d 1 \times 4 a$ and $d \mid x 5 a$, are transcribed at high levels in the cichlids' pharyngeal jaw and anal fin. The $d l x$ paralogs emerge as excellent candidate genes for the development of evolutionary innovations in cichlids, although further functional analyses are necessary to elucidate their respective contribution.

Keywords: Distal-less homeobox gene, Molecular evolution, Cichlid fishes, Teleost fishes, Positive selection, Differential selection, Gene duplication, dN/dS

\section{Background}

Teleost fishes (Teleostei) are among the most diverse lineages on Earth and with nearly 30,000 species the most species-rich vertebrate group. This is in stark contrast to the more basal non-teleost ray-finned fishes that are characterized by small numbers of species. A causal explanation for this discrepancy in speciation rates between the derived Teleostei and the non-teleost ray-finned fishes might be the fish-specific genome duplication (FSGD) that

\footnotetext{
* Correspondence: evelinetdiepeveen@gmail.com

Zoological Institute, University of Basel, Vesalgasse 1, 4051 Basel, Switzerland
}

occurred in the ancestor of modern teleosts ([1-4] and references therein). It has been hypothesized that the FSGD has laid down the genetic conditions necessary for the evolution of phenotypic diversity [5], although the exact causes of diversification of such a large clade are likely to be more complex and most probably also include other factors [6].

The Hox gene clusters, which evolved through both tandem and whole genome duplications, represent illustrative examples for the contribution of duplicated genes to morphological evolution across the animal kingdom (see e.g., [7-9]). Together with other homeotic genes, 
Hox genes play a crucial role in the development of the multicellular body plan (e.g., anterior-posterior patterning; [10]). Furthermore, Hox genes are known to be involved in the development of evolutionary novelties, such as walking limbs and the wings of insects [11-15]. It has been shown that different mechanisms such as cisregulatory evolution, changes in protein function and posttranscriptional regulation of the Hox genes contribute to morphological diversification (reviewed in e.g., $[8,15,16])$.

East African cichlid fishes show a remarkable level of phenotypic diversity between closely related species and constitute the most diverse adaptive radiations known [17-21]. Although several smaller radiations of cichlid fishes exist outside of Africa (e.g., in Central and South America), an astonishingly high number of cichlid species (close to 1900 species [22]) evolved in and around lakes Malawi, Victoria and Tanganyika in the last few million to several thousand years $[23,24]$. The various cichlid species differ in body shape, coloration, reproductive biology and mouth morphology [25-27] - traits which are thought to, at least partly, underlie the evolutionary success of cichlid fishes $[18,27,28]$. Furthermore, several morphological innovations are unique to cichlids or specific lineages thereof. The highly modified and morphological diverse pharyngeal jaw apparatus, for example, correlates with the diversity in foraging strategies exploited by the different cichlid species [27-29]. The occurrence of several color morphs within species, sexual color dimorphism and anal fin egg-spots are three characteristic features of the extremely speciesrich and mouthbrooding haplochromine lineage [30].

As a result of their great phenotypic diversity and high number of species, cichlid fishes provide an ideal set up to examine the genetic basis of rapid speciation, evolutionary innovations and adaptation [21,31-37]. An important strategy is the study of so-called candidate genes, i.e., genes with known functions in development in other organisms such as zebrafish. For example, it has been shown that csf1ra, which was identified as xanthophore marker in zebrafish $[38,39]$ is involved in the morphogenesis of the egg-spots of haplochromine cichlids [31]. Furthermore, species-specific jaw shapes of different cichlid species correlate with differences in early bmp4 expression patterns, a gene which has also the potential to change the mandibular morphology in zebrafish [40]. Many of these candidate genes belong to larger gene families such as the endothelin family of ligands and receptors that are putatively involved in the morphogenesis of the pharyngeal jaw apparatus and pigmentation [36], and the above mentioned Hox gene clusters [41].

Recently, Renz et al. [35] characterized seven distal-less homeobox $(d l x)$ genes and examined their expression patterns in the developing pharyngeal arches and/or pharyngeal teeth of the haplochromine cichlid Astatotilapia burtoni. The vertebrate $d l x$ genes are widely known for their crucial roles in the development (of components) of the nervous system, craniofacial skeleton and connective tissue and in the formation of appendages [reviewed in 42]. These functions seem to be conserved across a wide range of animal taxa. For example, the vertebrate $d l x$ genes are homologs of, and share several functions with, the single Distal-less ( $\mathrm{dll}$ ) gene of Drosophila [42]. Within vertebrates, the expression patterns of dlx homologs is similar in early development [35,42-45]. At the same time, $d l x$ genes have been implicated with evolutionary novelties such as the eyespots in various butterfly species [46-48], the insect antenna $[49,50]$ and the vertebrate craniofacial bones [51].

Phylogenetic analyses and the chromosomal arrangements of the vertebrate $d l x$ genes suggest that the extant $d l x$ repertoire has evolved by an initial tandem duplication, followed by two rounds of whole genome duplication in the lineage towards vertebrates and a third one in the lineage towards teleost fishes, the FSGD $[35,44,45,52]$. These duplication events resulted in multiple so-called $d l x$ clusters, in which two $d l x$ genes are located in a tail-to-tail arrangement on the respective chromosome. Linked $d l x$ genes are transcribed coincidently due to shared cisregulating elements in the intergenic regions $[35,42,43]$. Four of these $d l x$ clusters have been identified in teleost

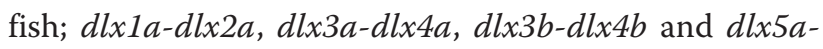
$d l x 6 a[43,44]$. Seven of these $d l x$ genes have been identified in the cichlid A. burtoni, where they are expressed in tissues that make up putative evolutionary innovations [35].

Here, we analyzed the $d l x$ repertoire and diversity in detail in a phylogenetically representative set of 23 East African cichlid species in order to study the molecular evolution of this prominent developmental gene family. To this end, we first performed phylogenetic comparisons of the dlx proteins including the sixty amino acids long homeobox domain in a range of teleost fishes in combination with blast searches of these sequences against the draft genomes of four cichlid species. Teleost and cichlid-specific phylogenies were examined to compare the rate of evolution of both between and within $d l x$ gene trees. Several studies have shown that loci putatively involved in evolutionary innovations are characterized by adaptive protein evolution in cichlids $[31,36,53]$. Therefore, all loci were screened for elevated rates of protein evolution by means of $d_{\mathrm{N}} / d_{\mathrm{S}}$ analyses. Our analyses indicate the presence of $d l x 3 a$ in cichlids and that the $d l x$ repertoire of cichlid fishes is shaped by differential selection pressures and rates of evolution, with signs of positive selection on specific sites in $d l x 1 a, d l \times 2 a, d l \times 3 a$ and $d l \times 4 b$.

\section{Methods}

\section{Dlx protein sequence comparison in teleost fishes}

The sequences of nine dlx proteins (i.e., dlx $1 \mathrm{a}, \mathrm{dl} \times 2 \mathrm{a}, \mathrm{dl} \times 2 \mathrm{~b}$, dlx3a, dlx3b, dlx4a, dlx4b, dlx5a and dlx6a) of seven teleost 
species (i.e., zebrafish (Danio rerio), Atlantic cod (Gradus morhua), three-spined stickleback (Gasterosteus aculeatus), spotted green pufferfish (Tetraodon nigroviridis), Japanese pufferfish (Takifugu rubripes), Japanese medaka (Oryzias latipes) and Nile Tilapia (Oreochromis niloticus)) were obtained from Ensemble (release 68, July 2012; see Additional file 1 for accession numbers). $D l x 2 b$ was excluded from all further analyses, due to its lineagespecific loss in percomorphs, to which all studied species belong except D. rerio and G. morhua (see [35]). Sequences were aligned with Tcoffee [54,55], ambiguous sites were removed and tblastx searches were performed to determine dlx protein sequences in the draft cichlid genomes of Astatotilapia burtoni, Neolamprologus brichardi and Pundamilia nyererei (BROAD Institute, unpublished data; see Additional file 1 for scaffold numbers). To determine the rate of evolution for each of the dlx proteins, phylogenetic analyses were performed in PAUP* 4.0 [56] under parsimony settings and the number of amino acid changes was obtained. D. rerio or G. morhua was used as outgroup species and bootstrap analyses with 100 replicates were conducted to test the robustness of the obtained topologies. Next, the sixty amino acids long homeobox domain was extracted from the sequences and aligned to the homeobox domain of the single Distal-less $(\mathrm{Dll})$ gene of Drosophila melanogaster [Ensemble: FBgn0000157] in Geneious 5.6 [57] for closer inspection of the conservation of the domain and to identify gene-specific substitutions.

\section{Cichlid samples and genomic DNA sequencing}

White muscle and/or fin clip samples were collected during fieldwork in Zambia in 2007 and 2008 using a standard operating procedure described in [29]. In total 23 Lake Tanganyikan cichlid species were included in this study (Additional file 2). Genomic DNA was extracted following a standard Proteinase K protocol [58]. Cichlidspecific PCR primers were designed based on available and/or draft genomic and transcriptomic cichlid sequences, which were identified by tblastx searches of publicly available $d l x$ sequences from other teleost species (see Additional file 1 for species and accession numbers). This was done for eight $d l x$ loci: $d l x 1 a, d l \times 2 a, d l \times 3 a, d l \times 3 b$, $d l x 4 a, d l x 4 b, d l x 5 a$ and $d l x 6 a$ (see Additional file 3 for primer sequences). Standard PCR reactions, purification steps and sequencing reactions were set up and performed as described elsewhere [36]. PCR products of the partially sequenced loci were visualized with GelRed (Biotium) on a $1.5 \%$ agarose gel and sequenced on a $3130 x l$ capillary sequencer (Applied Biosystems). Partial sequences were aligned and visually inspected using Codon Code Aligner 3.7.1 (CodonCode Corporation, Dedham, MA). Exon/intron boundaries were determined by homology comparisons with the sequences from the other teleost species. All generated cichlid $d l x$ sequences have been deposited into GenBank [GenBank: KC285366KC285546] (Additional file 2).

\section{Phylogenetic analyses of cichlid samples}

Individual gene trees were constructed using maximum likelihood in PAUP* 4.0 [56] and Bayesian Inference in MrBayes 3.2 [59,60]. The best-fitting model of nucleotide substitution was determined with the corrected Akaike information criteria and likelihood ratio tests conducted in jModeltest 0.1.1 [61,62]. Bootstrap analyses with 100 replicates were performed in PAUP* and MrBayes was run for 10.500 .000 generations. Oreochromis tanganicae was used as outgroup (see e.g., [63]). Phylogenetic analysis of a concatenated dataset of $9.2 \mathrm{~kb}$ was performed as described above in PAUP* to generate a common input tree file (treeBASE submission 14433) for the subsequent analyses.

Coding sequence data of the 23 cichlid species (treeBASE submission 14433) was assessed with both site- and branchsite models as implemented in the program Codeml of the software package PAML (Phylogenetic Analysis by Maximum Likelihood) $4.3[64,65]$. The following parameters were estimated for all eight $d l x$ datasets under different models: the nonsynonymous/synonymous substitution rate ratio, $\omega$, the proportion of sites assigned to an $\omega$ category, $p_{0,1,2}$, and the $p$ and $q$ parameters of the $\beta$ distribution. Tests of positively selected sites were conducted by performing Likelihood Ratio Tests (LRT) of the following model comparisons: M1a (Nearly Neutral) with M2a (Positive Selection), M7 ( $\beta)$ with M8 $\left(\beta \& \omega_{\mathrm{s}} \geq 1\right)$, and M8a $\left(\beta \& \omega_{\mathrm{s}}=1\right)$ with M8. The comparison between M0 (one-ratio) and M3 (discrete) was used as a test of variable $\omega$ among sites. The naive empirical Bayes (NEB; $[66,67]$ ) and the Bayes empirical Bayes (BEB; [68]) criteria were used to calculate the posterior probabilities for site classes and the BEB was used to identify sites under positive selection when the LRT was significant. To test whether the $d l x$ genes evolved under non-neutral evolution in specific lineages a LRT between the null model $\left(\omega_{\mathrm{s}}=1\right)$ and the alternative model $\left(\omega_{\mathrm{s}} \geq 1\right)$ was performed in the branchsite analyses. Branches of interest, or so-called foreground branches, were chosen based on the results of the phylogenetic analyses and branch tests performed in Hyphy ([69], following [36]).

Additional tests of positive selection on the partial $d l x$ sequences were performed with the Sitewise Likelihood Ratio estimation of selection program (SLR; [70]) v1.3. The common input tree file was used (see above) and the significance level was set to $95 \%$.

Amino acid substitutions were screened for possible effect on protein function with the program SIFT (Sorting Intolerant from Tolerant; [71]. 


\section{Results}

\section{Dlx protein sequence comparison in teleost fishes}

The tblastx searches of the teleost dlx proteins resulted in the retrieval of eight $d l x$ genes in all four cichlid species. Furthermore, the genomic locations of these $d l x$ loci (Additional file 1) indicate that four $d l x$ clusters are present in the cichlid lineage: $d l x 1 a-d l \times 2 a$; $d l \times 3 a-d l \times 4 a$; $d l x 3 b-d l x 4 b$ and $d l x 5 a-d l x 6 a$. All other teleost species examined contain this full set of genes, except zebrafish, in which $d l x 3 a$ could not be located, and medaka, in which $d l x 4 b$ is missing, as previously noted [35,44,45]. Interestingly, in contrast to Renz et al. [35] we do find evidence for the existence of $d l x 3 a$ in cichlids, including A. burtoni (Figure 1, Additional file 4).

The sixty amino acid long homeobox domain of the eight teleost $\mathrm{dlx}$ proteins are highly conserved among teleost fish and even between teleosts and the single Dll protein of D. melanogaster (Additional file 4). Despite the high level of conservation, several locus-specific amino acid substitutions are present in the paralogs, making it possible to distinguish between individual dlx homeobox domains.

Phylogenetic analyses of the dlx protein sequences were performed to examine the rate of evolution of the $\mathrm{dlx}$ paralogs in teleost fishes. The overall and relative longest trees were found for $d l x 4 b$ and $d l x 3 a$, while for $d l x 1 a$ and $d l x 6 a$ the shortest tree lengths were observed (Figure 1 and Table 1). Typically the longest branches were observed in the two basal species D. rerio and G. morhua. Interestingly, relatively long branch lengths for the branch towards the four cichlid species were observed for $d l x 3 a$ and $d l x 6 a$, indicting elevated rates of molecular evolution. The opposite scenario was observed in the overall more conserved dlx1a and dlx5a proteins. To study these effects in more detail cichlid specific gene trees were constructed.

The rate of $d l x$ gene evolution in East African cichlid fishes To reconstruct the molecular evolutionary history of the $d l x$ homologs in East African cichlid species, we determined the rate of evolution and the signatures of selection
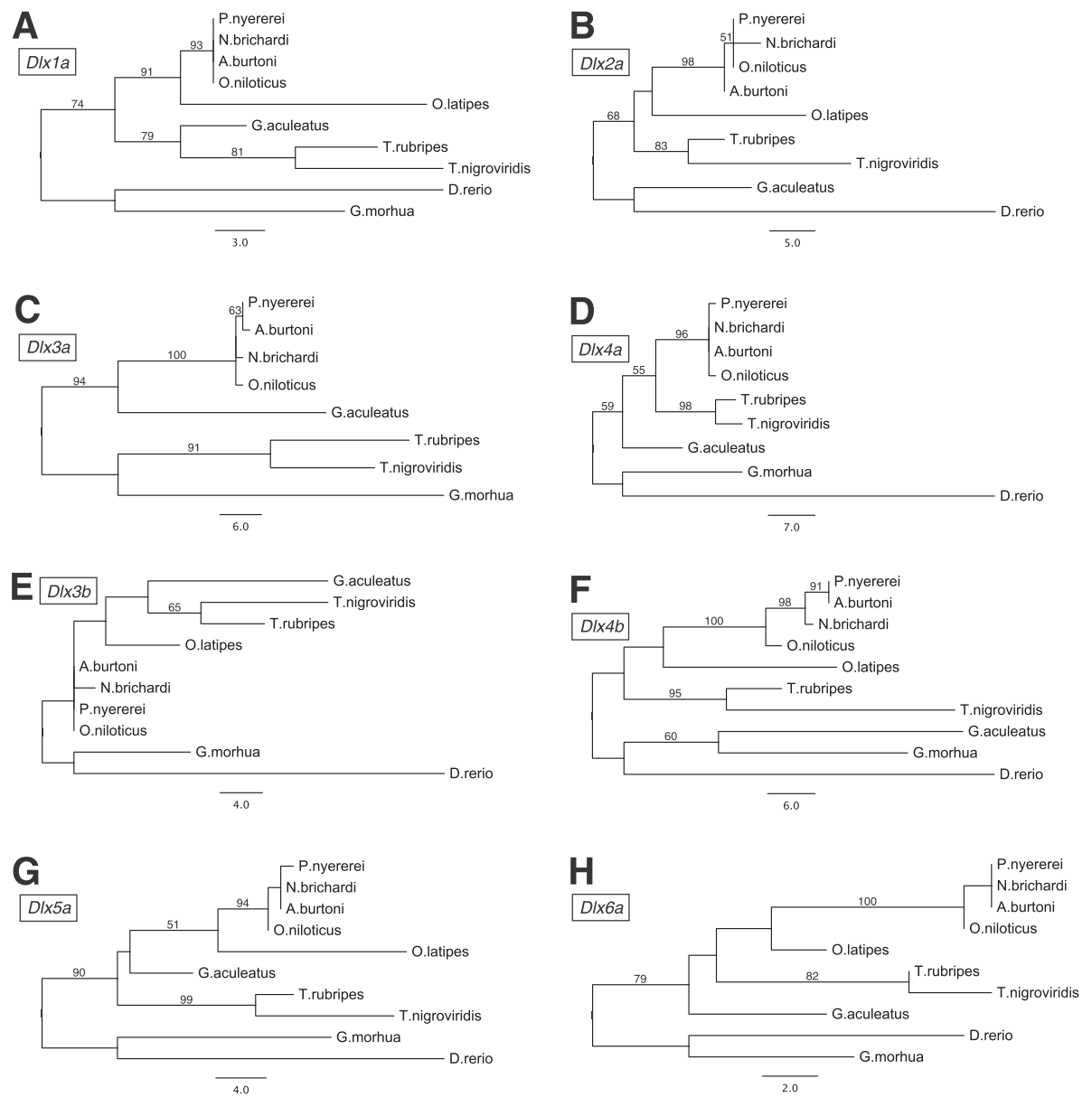

Figure 1 Maximum Likelihood phylogenetic hypotheses for the eight dlx paralogs in teleost fishes. (A) Dlx1a (254 amino acids (aa)). (B) Dlx2a (276 aa). (C) Dlx3a (307 aa). (D) Dlx4a (259 aa). (E) Dlx3b (283 aa). (F) Dlx4b (257 aa). (G) Dlx5a (285 aa). (H) Dlx6a (247 aa). Bootstrap probabilities (PAUP*) above $50 \%$ are shown. 
Table 1 Overall and relative tree lengths of teleost protein phylogenies

\begin{tabular}{cccc}
\hline dlx paralog & $\begin{array}{c}\text { Length } \\
\text { (\# amino acids) }\end{array}$ & $\begin{array}{c}\text { Tree length } \\
\text { (absolute) }\end{array}$ & $\begin{array}{c}\text { Tree length } \\
\text { (relative) }\end{array}$ \\
\hline dlx1a & 254 & 93 & 0.366 \\
dlx2a & 276 & 122 & 0.442 \\
dlx3a & 307 & 179 & 0.583 \\
dlx3b & 283 & 108 & 0.382 \\
dlx4a & 259 & 123 & 0.475 \\
dlx4b & 257 & 222 & 0.864 \\
dlx5a & 285 & 125 & 0.439 \\
dlx6a & 247 & 56 & 0.227 \\
\hline
\end{tabular}

pressure in a phylogenetically representative set of 23 species. The gene trees of the obtained partial cichlid $d l x$ sequences resulted in various polytomies (Additional file 5), probably due to the limited size of some of the datasets (minimum of $0.7 \mathrm{~kb}$ ). Although for each gene tree specific branches were observed with relative long branches, there is not a particular species or clade that has evolved under faster rates of evolution in all of the $d l x$ loci examined. Interestingly, three branches have relative long branch lengths in multiple topologies: the branch towards the Lamprologini $(d l \times 2 a, d l \times 4 a$ and $d l \times 5 a)$, C. leptosoma (dlx3b, $d l \times 4 a$ and $d l \times 5 a$ ) and C. furcifer (dlxla and $d l x 6 a$ ). The relative tree lengths (Additional file 5 and Table 2) of these gene trees reveal similar results as the teleost protein trees, with $d l \times 4 b$ and $d l \times 3 a$ evolving fastest and $d l x 1 a$ and $d l x 6 a$ evolving more slowly.

Observed signatures of selection pressure in cichlid $d / x$ loci To investigate signatures of selection pressure in the $d l x$ loci, we performed detailed analyses of the $d_{\mathrm{N}} / d_{\mathrm{S}}$ ratios. Maximum likelihood parameter estimations for $\omega, p_{0,1,2}$ and $p$ and $q$ under different evolutionary models can be found in Table 3 for all eight $d l x$ loci. Estimations of $\omega$ under the M0 model suggest that the $d l x$ genes evolved under purifying selection with $\omega$ ranging from 0.0001 $(d l \times 5 a)$ to $0.457(d l \times 2 a)$. A small proportion of sites,

Table 2 Overall and relative tree lengths of cichlid $d / x$ gene trees

\begin{tabular}{|c|c|c|c|}
\hline dlx paralog & $\begin{array}{c}\text { Length } \\
\text { (\# base pairs) }\end{array}$ & $\begin{array}{c}\text { Tree length } \\
\text { (absolute) }\end{array}$ & $\begin{array}{c}\text { Tree length } \\
\text { (relative) } 10^{-4}\end{array}$ \\
\hline dlx1a & 737 & 0.036 & 0.483 \\
\hline $\mathrm{dl} \times 2 \mathrm{a}$ & 1371 & 0.094 & 0.684 \\
\hline $\mathrm{dl} \times 3 \mathrm{a}$ & 666 & 0.061 & 0.910 \\
\hline$d l \times 3 b$ & 1972 & 0.120 & 0.609 \\
\hline$d \mid x 4 a$ & 1166 & 0.104 & 0.892 \\
\hline$d l \times 4 b$ & 722 & 0.068 & 0.937 \\
\hline$d l \times 5 a$ & 1538 & 0.093 & 0.607 \\
\hline dlx6a & 1710 & 0.093 & 0.542 \\
\hline
\end{tabular}

0.00001-24.2\%, was estimated to have evolved neutrally $(\omega=1)$ under the M1a model. By using models that allow $\omega$ to vary among sites, $0.7-12.3 \%$ of sites was detected with $\omega>1$ in $d l x 1 a, d l x 2 a, d l x 3 a, d l x 4 b$ and $d l x 6 a$. Overall, most sites are estimated to have evolved under purifying selection, with highest proportions found in $d l x 3 b, d l \times 4 a$ and $d l x 5 a$.

Likelihood ratio tests of the subsequent model comparisons (Table 4) resulted in the rejection of the null models in only the following comparisons per loci: $d l x 1 a$ (M8a-M8), $d l x 2 a$ (all four comparisons), dlx3a (M0-M3; M8a-M8) and $d l x 4 b$ (all four comparisons). Positively selected sites were detected with the BEB in $d l x 2 a$ (5 sites), $d l \times 3 a$ ( 1 site) and $d l \times 4 b$ ( 3 sites; see Table 4, Figure 2$)$. The less constraining analyses with the NEB resulted in two more putative positively selected sites in $d l x 1 a$ (1) and $d l x 2 a$ (1; Figure 2). Fewer positively selected sites were identified by the SLR analyses for $d l x 2 a$ (position: 36; significance: 99\%), $d l x 3 a(37,157 ; 99 \%, 95 \%)$ and $d l x 4 b$ (145; 99\%).

None of the performed LTRs of the branch-site analyses were significant $(1 \geq p \geq 0.20)$ indicating that although the $\omega$ ratios do vary among sites (see above), the $\omega$ ratios do not vary significantly among lineages.

\section{Amino acid substitutions and their predicted effect on function}

Next, the individual amino acid substitutions were examined in more detail. The total protein length and the number of amino acid substitutions per locus are shown in Table 5 (see also Figure 2 and Additional file 6). A relative large number of substitutions was observed in $d l \times 2 a$ (13), $d l x 3 a$ (16) and $d l x 4 b(10)$, while in $d l \times 5 a$ no substitution was found. Most of the amino acid substitutions are species-specific (i.e., observed in a single species), although lineage-specific substitutions were observed for the lamprologines $(d l x 2 a, d l x 3 a, d l x 4 b)$, ectodines $(d l x 2 a)$ and haplochromines $(d l x 2 a, d l x 3 a)$. None of the observed amino acid substitutions have a predicted effect on the protein functions (see Table 5), although two substitutions were observed in the homeobox domain of $d l \times 2 a$ (Figure 2).

\section{Selection regimes on the $d l x$ clusters}

It is known that the paired members of each of the four $d l x$ clusters (Additional file 4) are transcribed concurrently [35,42-45]. To characterize if the members of the same $d l x$ cluster evolved at similar rates and under similar selection regimes, we had a closer inspection of these paired genes. First, the teleost dlx protein and cichlid gene trees show that overall and relative tree lengths (or the rate of evolution) differ between the two genes within a cluster. Loci with the highest (dlx3a: 0.583/0.910 and $d l x 4 b: 0.864 / 0.937)$ or the smallest (dlx1a: $0.366 / 0.483$ and $d l x 6 a: 0.227 / 0.542)$ tree lengths are never observed 
Table 3 Site model parameter estimates generated by the CodeML analyses for the eight dlx paralogs

\begin{tabular}{|c|c|c|c|c|c|c|c|}
\hline \multirow[t]{2}{*}{ Locus } & \multicolumn{7}{|c|}{ Parameter estimates under different models } \\
\hline & Mo (one ratio) & M1a (neutral) & M2a (selection) & M3 (discrete) & M7 (ß) & M8 (ß \& $\omega)$ & M8a $\left(ß \& \omega_{s}=1\right)$ \\
\hline \multirow[t]{3}{*}{$d \mid x 1 a$} & $\omega=0.111$ & $p_{0}=0.940, \omega_{0}=0$ & $p_{0}=0.980, \omega_{0}=0$ & $p_{0}=0.640, \omega_{0}=0$ & $p=0.005, q=0.049$ & $p=0.005, q=22.651$ & $p=1.333, q=24.671$ \\
\hline & & $p_{1}=0.060, \omega_{1}=1$ & $p_{1}=0, \omega_{1}=1$ & $p_{1}=0.340, \omega_{1}=0$ & & $p_{0}=0.980$ & $p_{0}=0.936$ \\
\hline & & & $p_{2}=0.020, \omega_{2}=12.580$ & $p_{2}=0.020, \omega_{2}=12.580$ & & $p_{1}=0.020, \omega=12.580$ & $p_{1}=0.065, \omega=1$ \\
\hline \multirow[t]{3}{*}{$d \mid x 2 a$} & $\omega=0.457$ & $p_{0}=0.833, \omega_{0}=0$ & $p_{0}=0.879, \omega_{0}=0$ & $p_{0}=0, \omega_{0}=0$ & $p=0.005, q=0.011$ & $p=0.005, q=0.046$ & $p=0.005, q=12.618$ \\
\hline & & $p_{1}=0.167, \omega_{1}=1$ & $p_{1}=0.110, \omega_{1}=1$ & $p_{1}=0.986, \omega_{1}=0.081$ & & $p_{0}=0.989$ & $p_{0}=0.833$ \\
\hline & & & $p_{2}=0.010, \omega_{2}=18.903$ & $p_{2}=0.014, \omega_{2}=16.629$ & & $p_{1}=0.011, \omega=18.053$ & $p_{1}=0.167, \omega=1$ \\
\hline \multirow[t]{3}{*}{$d 1 \times 3 a$} & $\omega=0.320$ & $p_{0}=0.758, \omega_{0}=0$ & $p_{0}=0.921, \omega_{0}=0$ & $p_{0}=0.877, \omega_{0}=0$ & $p=0.005, q=0.017$ & $p=0.015, q=0.088$ & $p=0.005, q=13.826$ \\
\hline & & $p_{1}=0.242, \omega_{1}=1$ & $p_{1}=0.034, \omega_{1}=1$ & $p_{1}=0.120, \omega_{1}=2.099$ & & $p_{0}=0.032$ & $p_{0}=0.758$ \\
\hline & & & $p_{2}=0.045, \omega_{2}=4.451$ & $p_{2}=0.003, \omega_{2}=11.657$ & & $p_{1}=0.032, \omega=5.096$ & $p_{1}=0.242, \omega=1$ \\
\hline \multirow[t]{3}{*}{$d 1 \times 3 b$} & $\omega=0.047$ & $p_{0}=1, \omega_{0}=0.047$ & $p_{0}=1, \omega_{0}=0.047$ & $p_{0}=0.243, \omega_{0}=0.047$ & $p=4.896, q=99.00$ & $p=4.895, q=99.00$ & $p=4.895, q=99.00$ \\
\hline & & $p_{1}=1 \mathrm{E}-5, \omega_{1}=1$ & $p_{1}=0, \omega_{1}=1$ & $p_{1}=0.262, \omega_{1}=0.047$ & & $p_{0}=1$ & $p_{0}=1$ \\
\hline & & & $p_{2}=0, \omega_{2}=1$ & $p_{2}=0.495, \omega_{2}=0.047$ & & $p_{1}=1 \mathrm{E}-5, \omega=1$ & $p_{1}=1 \mathrm{E}-5, \omega=1$ \\
\hline \multirow[t]{3}{*}{$d \mid x 4 a$} & $\omega=0.050$ & $p_{0}=1, \omega_{0}=0.050$ & $p_{0}=1, \omega_{0}=0.050$ & $p_{0}=0.216, \omega_{0}=0.050$ & $p=5.267, q=99.00$ & $p=5.266, q=99.00$ & $p=0.137, q=2.305$ \\
\hline & & $p_{1}=1 \mathrm{E}-5, \omega_{1}=1$ & $p_{1}=0, \omega_{1}=1$ & $p_{1}=0.384, \omega_{1}=0.050$ & & $p_{0}=1$ & $p_{0}=1$ \\
\hline & & & $p_{2}=0, \omega_{2}=1$ & $p_{2}=0.400, \omega_{2}=0.050$ & & $p_{1}=1 \mathrm{E}-5, \omega=1$ & $p_{1}=1 \mathrm{E}-5, \omega=1$ \\
\hline \multirow[t]{3}{*}{$d \mid x 4 b$} & $\omega=0.259$ & $p_{0}=0.872, \omega_{0}=0$ & $p_{0}=0.993, \omega_{0}=0.090$ & $p_{0}=0, \omega_{0}=0$ & $p=0.005, q=0.042$ & $p=9.871, q=99.00$ & $p=0.005, q=30.817$ \\
\hline & & $p_{1}=0.128, \omega_{1}=1$ & $p_{1}=0, \omega_{1}=1$ & $p_{1}=0.993, \omega_{1}=0.090$ & & $p_{0}=0.993$ & $p_{0}=0.872$ \\
\hline & & & $p_{2}=0.007, \omega_{2}=12.858$ & $p_{2}=0.007, \omega_{2}=12.858$ & & $p_{1}=0.007, \omega=12.859$ & $p_{1}=0.128, \omega=1$ \\
\hline \multirow[t]{3}{*}{$d 1 \times 5 a$} & $\omega=1 \mathrm{E}-4$ & $p_{0}=1, \omega_{0}=0$ & $p_{0}=1, \omega_{0}=0$ & $p_{0}=0.424, \omega_{0}=0$ & $p=0.005, q=1.294$ & $p=0.005, q=99.00$ & $p=0.005, q=44.274$ \\
\hline & & $p_{1}=1 \mathrm{E}-5, \omega_{1}=1$ & $p_{1}=0, \omega_{1}=1$ & $p_{1}=0.420, \omega_{1}=0$ & & $p_{0}=1$ & $p_{0}=1$ \\
\hline & & & $p_{2}=0, \omega_{2}=1$ & $p_{2}=0.156, \omega_{2}=0$ & & $p_{1}=1 \mathrm{E}-5, \omega=1$ & $p_{1}=1 \mathrm{E}-5, \omega=1$ \\
\hline \multirow[t]{3}{*}{$d 1 \times 6 a$} & $\omega=0.029$ & $p_{0}=0.967, \omega_{0}=0$ & $p_{0}=0.992, \omega_{0}=0$ & $p_{0}=0.899, \omega_{0}=0$ & $p=0.066, q=1.696$ & $p=0.005, q=2.390$ & $p=0.005, q=1.672$ \\
\hline & & $p_{1}=0.033, \omega_{1}=1$ & $p_{1}=0, \omega_{1}=1$ & $p_{1}=0.093, \omega_{1}=0$ & & $p_{0}=0.992$ & $p_{0}=0.967$ \\
\hline & & & $p_{2}=0.008, \omega_{2}=6.336$ & $p_{2}=0.008, \omega_{2}=6.336$ & & $p_{1}=0.008, \omega=6.336$ & $p_{1}=0.033, \omega=1$ \\
\hline
\end{tabular}

Note: $\mathrm{p}_{0-2}$ are the proportions of sites assigned to an $\omega$ category or to a beta distribution with $\mathrm{p}$ and $\mathrm{q}$ as parameters. $\omega$ ratios greater than one and their corresponding proportions are depicted in bold. 
Table 4 Likelihood ratio test (LTR) statistics of site model comparisons for dIx1a, dIx2a, dIx3a and dIx4b

\begin{tabular}{|c|c|c|c|c|}
\hline Locus & Test & LRT $(2 \Delta l)$ & $\mathrm{p}$ & Selected sites (BEB) \\
\hline \multirow[t]{4}{*}{$d \mid x 1 a$} & M0 vs M3 & 8.416 & 0.077 & - \\
\hline & M1a vs M2a & 3.396 & ns & - \\
\hline & M7 vs M8 & 3.680 & ns & - \\
\hline & M8a vs M8 & 5.084 & 0.012 & - \\
\hline \multirow[t]{4}{*}{$d \mid x 2 a$} & M0 vs M3 & 70.438 & $<0.001$ & - \\
\hline & M1a vs M2a & 39.198 & $<0.001$ & 36A, $48 \mathrm{~T}, 255 \mathrm{l}$ \\
\hline & M7 vs M8 & 43.093 & $<0.001$ & 36A, $48 \mathbf{T}, 195 A, 254 A, 255 ।$ \\
\hline & M8a vs M8 & 39.168 & $<0.001$ & See M7 vs M8 comparison \\
\hline \multirow[t]{4}{*}{$d \mid x 3 a$} & M0 vs M3 & 12.605 & 0.013 & - \\
\hline & M1a vs M2a & 3.858 & ns & - \\
\hline & M7 vs M8 & 4.258 & ns & - \\
\hline & M8a vs M8 & 3.872 & 0.025 & 375 \\
\hline \multirow[t]{4}{*}{$d 1 \times 4 b$} & M0 vs M3 & 39.110 & $<0.001$ & - \\
\hline & M1a vs M2a & 16.940 & $<0.001$ & $48 Q, 135 A, 145 \mathrm{~T}$ \\
\hline & M7 vs M8 & 17.367 & $<0.001$ & $48 Q, 135 A, 145 \mathrm{~T}$ \\
\hline & M8a vs M8 & 16.931 & $<0.001$ & See $M 7$ vs M8 comparison \\
\hline
\end{tabular}

LRT values, $p$-values and positively selected sites identified by the BEB ( $p<0.01$ (in bold) and $\mathrm{p}<0.05$ (in italic); CodeML) are shown.

within the same cluster. Furthermore, the mode of selection seems to differ between members of the same $\mathrm{dlx}$ clusters as well. While strong purifying selection was observed for $d l \times 3 b, d l \times 4 a$ and $d l \times 5 a$, their paired cluster members $d l x 4 b, d l x 3 a$ and $d l x 6 a$ show sign of elevated $\omega$-values. A notable exception to this observation is the $d l x 1 a-d l x 2 a$ cluster. For both genes a proportion of sites was found with elevated $\omega$-values (note that the proportion is considerably bigger for $d l x 2 a$ ). These observations indicate that although clusters are transcribed concurrently, selection seems to act on the individual gene level rather than on the level of the $d l x$ gene clusters. Also the observed patterns are not in concordance with the two groups of homeobox domains that emerged from the initial tandem duplication (see [52] and Additional file 4).

\section{Discussion}

In this work, we present a detailed evolutionary characterization of the $d l x$ gene repertoire in East African cichlid fishes. Previously, Renz et al. [35] studied the embryonic expression patterns of $d l x$ genes in cichlids and showed that they are expressed in e.g., the developing jaw apparatus and anal fin, tissues that contribute to two putative evolutionary innovations: the pharyngeal jaw and the egg-spots on the anal fin of the cichlid A. burtoni. Here, we study the molecular evolution of $d l x$ genes in a representative set of 23 East African cichlid species. We performed comparative phylogenetic analyses and detailed screens of nonsynonymous-synonymous substitution rate ratios to determine the selective pressure acting upon these candidate genes for evolutionary novelties in cichlid fishes.

\section{Dlx3a did not get lost in the cichlid lineage}

Our phylogenetic analyses of dlx proteins extends previous analyses (e.g., [35]) by the inclusion of cod [72] and four different cichlid species (i.e., O. niloticus, $N$. brichardi, A. burtoni and P. nyererei; BROAD Institute). Although our results agree with most of the available hypotheses on the evolutionary loss of $d l x$ genes in specific teleost lineages (i.e., $d l x 3 a$ in zebrafish and $d l x 4 a$ in medaka), we did detect $d l x 3 a$ in cichlids and thus refute the cichlid-specific gene loss hypothesis of $d l x 3 a$ put forward by Renz et al. [35]. Not only were we able to locate this gene in all four cichlid genomes examined (Additional file 1), we also gathered partial gene sequences for this locus in all 23 cichlid species included (Additional files 4 and 6). Furthermore, in-house tblastx searches of this newly identified paralog against preliminary cichlid EST libraries (BROAD Institute, unpublished data) resulted in multiple hits, providing proof of its expression in - at least - Astatotilapia burtoni, Oreochromis niloticus and Metriaclima zebra.

\section{Selection on $d l x$ paralogs in relation to gene duplication events}

Gene-wide estimates of the $d_{\mathrm{N}} / d_{\mathrm{S}}$ ratios indicate that all loci evolved under purifying selection $(\omega<1)$, indicating strong selection against deleterious mutations, commonly observed in functional proteins. Additional analyses of individual codons indicate that the sequenced regions of $d l x 3 b, d l \times 4 a$ and $d l \times 5 a$ evolved under purifying selection, while positive selection acting on specific codons was detected for a small proportion of sites (i.e., up to $12 \%$ ) for $d l x 1 a, d l \times 2 a, d l \times 3 a$ and $d l x 4 b$ (i.e., a smaller number of positively selected sites was found with more stringent SLR analyses for $d l \times 2 a, d l \times 3 a$ and $d l \times 4 b$ ). Plausible reasons for the excess of nonsynonymous mutations in these loci are either lowered functional constraints or directional selection, as Sumiyama and colleagues suggested for $D l x 7$ in mouse [73]. Different modes of selection are thus found to have acted on the $d l x$ paralogs in cichlids after the genome duplication events.

Differential selection after gen(om)e duplication is a commonly observed phenomenon and is associated with the fate of the gene duplicates i.e., non-, sub- or neofunctionalization. Sub- and neofunctionalization are adaptive processes by which either spatial or temporal partitioning of the ancestral function or the evolution of complete new functions take place [5,74-76]. While ancestral functions can be maintained by retaining the protein sequences and preventing deleterious mutations through purifying selection, relaxed selection on the other duplicate can lead to the introduction of mutations and subsequent divergence 
$[5,75,76]$. Most of these changes are deleterious and are followed by the loss of the gene over time (i.e., nonfunctionalization). On rare occasions the mutations can lead to an altered function of the protein (i.e., neofunctionalization; change within the protein) or altered expression pattern (subfunctionalization; change in regulatory regions), which can be characterized by elevated $\omega$ values and the maintenance of the mutations results in divergence of the two duplicates.
Many studies have focused on duplicated genes in relation to divergence of duplicates (see e.g., [77-80] and references therein). An interesting case of subfunctionalization was described in leaf-eating Colobine monkeys, in which the pancreatic ribonuclease gene (RNASE1), necessary to digest its specialized diet, was duplicated $[81,82]$. Although the two gene-products are used in the same process (i.e., digestion of bacterial RNA), the duplicate gene shows many substitutions, while the ancestral locus 


$\begin{aligned} & \text { Table } 5 \text { Amino acid substitution and their predicted } \\
& \text { effect on function for the eight cichlid } \boldsymbol{d} \text { lx loci }\end{aligned}$
\begin{tabular}{lccc}
\hline Locus & $\mathbf{L}$ & S & E \\
\hline$d 1 \times 1 a$ & 144 & 1 & Tolerated \\
$d 1 \times 2 a$ & 255 & 13 & Tolerated \\
$d 1 \times 3 a$ & 222 & 16 & Tolerated \\
$d 1 \times 3 b$ & 160 & 4 & Tolerated \\
$d 1 \times 4 a$ & 96 & 1 & Tolerated \\
$d 1 \times 4 b$ & 167 & 10 & Tolerated \\
$d 1 \times 5 a$ & 271 & 0 & - \\
$d 1 \times 6 a$ & 236 & 2 & Tolerated
\end{tabular}

Length of obtained protein sequence (L), the number of amino acid substitutions $(\mathrm{S})$ and the predicted effect of the substitutions on the function generated by the SIFT analyses (E) are shown.

did not change [81]. Similar patterns of heterogeneity in amino acid substitutions or differential selection were also observed by Dermitzakis and Clark [83] between duplicates of several developmental gene families (e.g., Notch, Bmp and Hox9) in mouse and human. Interestingly, differential selection regimes acting on paralogs were also found in the murine $D l x 3-D l x 7$ cluster, with $D l x 7$ evolving more rapidly than $D l \times 3$ [73]. Our results of differential selection acting on the cichlid $d l x$ paralogs are thus comparable to previously studied cases of duplicated genes. We even detect a similar pattern as Sumiyama et al. [73], with $d l x 4 b$ evolving more rapidly than $d l \times 3 b$ (i.e., relative tree length 0.937 vs 0.609 ).

The adaptive protein evolution as observed in dlx1a, $d l \times 2 a, d l \times 3 a$ and $d l \times 4 b$ together with the evolutionary history of the gene family, could thus be a sign of possible new or altered functions of these $d l x$ paralogs in cichlids. Although we did not observe amino acid substitutions with predicted apparent effect on the protein function in our partial sequences, other mechanisms, such as cis-regulatory evolution might have altered the expression patterns after gene duplication. Gene expression analyses in cichlid and zebrafish indicate that clusters are often transcribed concurrently and that the $d l x d u-$ plicates exhibit overlapping expression patterns in particular during the development of brain and pharyngeal arches $[35,44,45]$. This co-expression of the $d l x$ clusters is controlled through intergenic cis-regulatory regions $[35,42,43]$. While mutations in these regions are expected to affect the expression of both paralogs, changes in the coding regions of the $d l x$ loci are likely to affect the individual $d l x$ locus' function, which could lead to neofunctionalization.

\section{Selection pressure on dlx paralogs in relation to evolutionary innovations}

We found an interesting pattern comparing our $d_{\mathrm{N}} / d_{\mathrm{S}}$ results with the expression patterns found by Renz et al.
[35] in relation to evolutionary novelties in cichlids. In the developing pharyngeal teeth and the anal fin $d l \times 3 b$, $d l x 4 a$ (not in anal fin) and $d l x 5 a$, the exact loci for which we found strong patterns of purifying selection, are expressed at high levels. Although this observation seems to contradict other cases in which candidate genes showed accelerated rates of protein evolution (see $[31,53,84])$, they do not stand alone (see e.g., [36]). It has been shown that minor changes in the complex genetic pathways underlying the development of morphological structures can lead to the evolution of novelties (see e.g., [85]). Furthermore, many cases of morphological adaptation are driven by cis-regulatory evolution (reviewed in [86]). Several intergenic cis-regulatory elements have been identified in the $d l x$ clusters in $A$. burtoni by Renz et al. [35], but the functional characterization in cichlids is yet to be performed. It is thus possible that only a small fraction of genes involved in the evolutionary novelties in cichlids show signs of adaptive evolution and that the three $d l x$ loci were co-opted for their ancestral functions.

According to Renz et al. [35], the five $d l x$ genes for which we found signatures of positive selection, are either not expressed at all or at low levels during pharyngeal teeth and anal fin development in the cichlid A. burtoni. Low levels of gene expression were observed for $d l x 2 a$ in the developing pharyngeal teeth in cichlids [35], while higher $d l x 2 a$ expression levels were observed in other teleost species $[33,44,45] . D l x 4 b$ and $d l x 6 a$ expression has previously been shown in the developing pharyngeal teeth of zebrafish and/or medaka $[44,45]$, but has not been observed in cichlids (yet). Furthermore, multiple $d l x$ genes, including loci with signatures of positive selection, appear to be expressed in the developing anal fin tissue at time points coinciding with egg-spot development in A. burtoni (E. Santos, personal communications). Therefore, it is likely that several $d l x$ paralogs, for which we found signs of positive selection, are involved in the development of evolutionary innovations in cichlids, in contrast to the initial findings of Renz et al. [35]. Future detailed and extended functional analyses should be conducted to elucidate their role in the development of these evolutionary important traits in cichlid fishes.

\section{Conclusions}

In this study, we provide an in depth molecular evolutionary analysis of the $d l x$ gene repertoire in teleost fishes. We located and generated partial sequences for $d l x 3 a$ in 23 East African cichlid species, refuting the hypothesis of Renz et al. [35] that $d l \times 3 a$ got lost in the cichlid lineage. Phylogenetic analyses of the teleost $d l x$ gene repertoire show that substantial differences exist in the rate of evolution among teleost $d l x$ paralogs. In addition, analyses of the nonsynonymous-synonymous 
substitution rates of the cichlid $d l x$ paralogs revealed strong differences in the selection pressure acting upon $d l x$ paralogs and cluster members. Although differential selection pressure after gene duplication is a putative sign of new or altered functions, we observed a link between the $d l x$ loci under strong purifying selection, in particular, and high expression levels in two cichlids' novelties; the pharyngeal jaw and anal fin. This indicates that other mechanisms than adaptive protein evolution are likely to be involved in the co-option of these genes. Furthermore, several (preliminary) studies found that at least three other $d l x$ paralogs, for which we found signs of positive selection, are actually expressed in the developing pharyngeal teeth and/or haplochromine anal fin. Hence, the $d l x$ paralogs appear as candidate genes for the development of evolutionary innovations in cichlids, although further functional analyses should elucidate the role of positive selection therein.

\section{Availability of supporting data}

The datasets supporting the results of this article are publicly available in the GenBank repository under accession numbers: KC285366-KC285546 and in the treeBASE repository under submission number 14433, http://purl.org/ phylo/treebase/phylows/study/TB2:S14433.

\section{Additional files}

\section{Additional file 1: Accession numbers and/or genomic location of} the teleost $d l x$ sequences.

Additional file 2: Specimen information and GenBank Accession numbers.

Additional file 3: Primer information and primer sequences. Additional file 4: Protein comparison of the teleost dlx homeobox domains. Depicted are the amino acid sequences of the homeobox

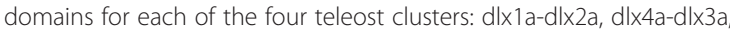
$d \mid \times 4 b-d l \times 3 b$ and dlx6a-dlx5a in comparison with the single Dll homeobox sequence (here depicted in duplo) of Drosophila melanogaster. Sequences can be divided in two groups; dlx1a, dlx4a, dl $\times 4 b$ and dlx6a versus $d|\times 2 a, d| \times 3 a, d \mid \times 3 b$ and $d \mid x 5 a$. The two sixty amino acid long homeobox domains of each cluster are depicted in separate boxes. The top graph displays the mean pairwise identity of all sequences (i.e., green $=100 \%$ identity and brown $\geq 30 \%$ identity). Numbers represent the amino acid position within the homeobox.

Additional file 5: Maximum likelihood gene trees based on 23 cichlid species for the eight $d \boldsymbol{d} \boldsymbol{x}$ loci. Bootstrap values (PAUP*) and Bayesian posterior probabilities (MrBayes) above 50\% are shown respectively above and below the branches. A color key for the ten studied cichlid lineages is given in the box below the figure. (a) D/x $1 a$ (737 base pairs (bp); TPM3uf model). Two major polytomies were recovered. The lamprologines cluster together with the Boulengerochromini, Bathybatini and the Cyphotilapiini. A. burtoni is found at the base with $O$. tanganicae. (b) D/x2a (1371 bp; HKY + I model). Polytomous tree with all members of the lineages Lamprologines, Ectodines, Haplochromines and Limnochromines recovered as monophyletic clades. (c) D/x3a (666 bp; HKY model). Polytomous tree, with only the Lamprologines recovered as monophyletic clade. (d) D/x4a (1166 bp; TPM3uf + I + G). Polytomous relationships were observed between multiple lineages, although most lineages are monophyletic except the Haplochromines (e) D/x3b (1972 bp; GTR + I + G). Moderately resolved tree. (f) D/x4b (722 bp; TPM3uf). Mostly polytomous relationships between species, except the Limnochromini and most members of the Lamprologines. (g) D/x5a (1538 bp; TIM2 + G). Basal polytomy divides ingroup species except G. permaxillaris, in two big clades. (h) D/x6a (1710 bp; TIM3 + G). Limnochromines, Lamprologines and Haplochromines recovered as monophyletic clades, although the relationships between lineages are largely polytomous.

Additional file 6: Four partially sequenced cichlid Dlx proteins. Depicted are the amino acid sequences of Astatotilapia burtoni $(\mathbf{a}, \mathbf{c}, \mathbf{d})$ and Ctenochromis horei (b). Secondary structure predictions were obtained from the PSIPRED server (http://bioinf.cs.ucl.ac.uk/psipred/). (a) Dl×3b. (b) DIx4a. (c) Dlx5a. (d) Dlx6a.

\section{Competing interest}

The authors declare that they have no competing interests.

\section{Authors' contributions}

ETD, FDK and WS conceived the study. FDK generated the data. ETD and FDK analyzed the data. ETD and WS wrote the paper. All authors read and approved the final manuscript.

\section{Authors' information}

ETD is a PhD student and FDK a master student in the group of WS. WS is a Professor of Zoology and Evolutionary Biology at the University of Basel. The research of his team focuses on the genetic basis of adaptation, evolutionary innovation and animal diversification of mainly the exceptionally diverse cichlid fishes.

\section{Acknowledgements}

We would like to express our gratitude to past and current members of the Salzburger lab for their contribution to sampling during fieldwork; to Brigitte Aeschbach and Nicolas Boileau for assistance during lab work; to Britta Meyer for advice on the phylogenetic analyses; to Emília Santos for help on designing the study and comments on earlier drafts of this manuscript; and to Richard Kluin for grammatical advice. We would also like to thank the BROAD institute for sharing unpublished cichlid genome sequence data with the community. The valuable suggestions of two anonymous reviewers greatly helped improving this manuscript. This study was supported by the Freiwillige Akademische Gesellschaft Basel (Dissertation support grant to ETD), European Research Council (Starting Grant "INTERGENADAPT" to WS) and the Swiss National Science Foundation (Grant 3100A0_122458 to WS).

Received: 9 May 2013 Accepted: 5 July 2013

Published: 17 July 2013

\section{References}

1. Meyer A, Van de Peer Y: From 2R to 3R: evidence for a fish-specific genome duplication (FSGD). Bioessays 2005, 27:937-945.

2. Volff JN: Genome evolution and biodiversity in teleost fish. Heredity 2005, 94:280-294.

3. Taylor JS, Braasch I, Frickey T, Meyer A, Van de Peer Y: Genome duplication, a trait shared by 22,000 species of ray-finned fish. Genome Res 2003, 13:382-390

4. Taylor JS, Van de Peer Y, Braasch I, Meyer A: Comparative genomics provides evidence for an ancient genome duplication event in fish. Phil Trans R Soc Lond B 2001, 356:1661-1679.

5. Ohno S: Evolution by gene duplication. New York: Springer Verlag; 1970

6. Santini F, Harmon LJ, Carnevale G, Alfaro ME: Did genome duplication drive the origin of teleosts? A comparative study of diversification in ray-finned fishes. BMC Evol Biol 2009, 9:194.

7. De Rosa R, Grenier JK, Andreevas T, Cook CE, Adoutte A, Akam M, Carroll SB, Balavoine G: Hox genes in brachiopods and priapulids and protostome evolution. Nature 1999, 399:772-776.

8. Lemons D, McGinnis W: Genomic evolution of Hox gene clusters. Science 2006, 313:1918-1922.

9. Ruddle FH, Bartels JL, Bentley KL, Kappen C, Murtha MT, Pendleton JW: Evolution of Hox genes. Annu Rev Genet 1994, 28:423-442.

10. Gehring WJ, Hiromi Y: Homeotic genes and the homeobox. Annu Rev Genet 1986, 20:147-173. 
11. Cohn MJ, Tickle C: Limbs: a model for pattern formation within the vertebrate body plan. Trends Genet 1996, 12:253-257.

12. Zakany J, Duboule D: The role of Hox genes during vertebrate limb development. Curr Opin Genet Dev 2007, 17:359-366.

13. Weatherbee SD, Nijhout HF, Grunert LW, Halder G, Galant R, Selegue J, Carroll SB: Ultrabithorax function in butterfly wings and the evolution of insect wing patterns. Curr Biol 1999, 9:109-115.

14. Warren RW, Nagy L, Selegue J, Gates J, Carroll SB: Evolution of homeotic gene regulation and function in flies and butterflies. Nature 1994, 372:458-461.

15. Pick L, Heffer A: Hox gene evolution: multiple mechanisms contributing to evolutionary novelties. Ann N Y Acad Sci 2012, 1256:15-32.

16. Pearson JC, Lemons D, McGinnis W: Modulating Hox gene functions during animal body patterning. Nat Rev Genet 2005, 6:893-904.

17. Seehausen $O$ : African cichlid fish: a model system in adaptive radiation research. Proc R SOC B 2006, 273:1987-1998.

18. Salzburger $W$ : The interaction of sexually and naturally selected traits in the adaptive radiations of cichlid fishes. Mol Ecol 2009, 18:169-185.

19. Kornfield I, Smith PF: African cichlid fishes: model systems for evolutionary biology. Annu Rev Ecol Syst 2000, 31:163-196.

20. Kocher TD: Adaptive evolution and explosive speciation: the cichlid fish model. Nat Rev Genet 2004, 5:288-298.

21. Santos ME, Salzburger W: How cichlids diversify. Science 2012, 338:619-621.

22. Turner GF, Seehausen O, Knight ME, Allender CJ, Robinson RL: How many species of cichlid fishes are there in African lakes? Mol Ecol 2001, 10:793-806.

23. Genner MJ, Seehausen O, Lunt DH, Joyce DA, Shaw PW, Carvalho GR, Turner GF: Age of cichlids: new dates for ancient lake fish radiations. Mol Biol Evol 2007, 24:1269-1282.

24. Verheyen E, Salzburger W, Snoeks J, Meyer A: Origin of the superflock of cichlid fishes from Lake Victoria, East Africa. Science 2003, 300:325-329.

25. Barlow GW: The cichlid fishes: nature's grand experiment in evolution. Cambridge: Perseus publishing; 2000.

26. Coulter GW: Lake Tanganyika and its life. Oxford: British Museum (Natural History) and Oxford University Press; 1991

27. Fryer G, lles TD: The cichlid fishes of the Great Lakes of Africa: their biology and Evolution. Edinburgh: Oliver \& Boyd; 1972:1-334.

28. Liem KF: Evolutionary strategies and morphological innovations: Cichlid pharyngeal jaws. Systematic Zoology 1973, 22:425-441

29. Muschick M, Indermaur A, Salzburger W: Convergent evolution within an adaptive radiation of cichlid fishes. Curr Biol 2012, 22:2362-2368.

30. Salzburger W, Mack T, Verheyen E, Meyer A: Out of Tanganyika: Genesis, explosive speciation, key-innovations and phylogeography of the haplochromine cichlid fishes. BMC Evol Biol 2005, 5:17

31. Salzburger W, Braasch I, Meyer A: Adaptive sequence evolution in a color gene involved in the formation of the characteristic egg-dummies of male haplochromine cichlid fishes. BMC Biol 2007, 5:51.

32. Roberts RB, Ser JR, Kocher TD: Sexual conflict resolved by invasion of a novel sex determiner in Lake Malawi cichlid fishes. Science 2009, 326:998-1001.

33. Fraser GJ, Hulsey CD, Bloomquist RF, Uyesugi K, Manley NR, Streelman JT: An ancient gene network is co-opted for teeth on old and new jaws. Plos Biol 2009, 7:e31.

34. Fraser GJ, Bloomquist RF, Streelman JT: A periodic pattern generator for dental diversity. BMC Biol 2008, 6:32.

35. Renz AJ, Gunter HM, Fischer JM, Qiu H, Meyer A, Kuraku S: Ancestral and derived attributes of the dlx gene repertoire, cluster structure and expression patterns in an African cichlid fish. EvoDevo 2011, 2:1.

36. Diepeveen ET, Salzburger W: Molecular characterization of two endothelin pathways in East African cichlid fishes. J Mol Evol 2011, 73:355-368.

37. Kuraku S, Meyer A: Genomic analysis of cichlid fish 'natural mutants'. Curr Opin Genet Dev 2008, 18:551-558.

38. Parichy DM, Ransom DG, Paw B, Zon LI, Johnson SL: An orthologue of the kit-related gene fms is required for development of neural crest-derived xanthophores and a subpopulation of adult melanocytes in the zebrafish, Danio rerio. Development 2000, 127:3031-3044.

39. Parichy DM, Turner JM: Temporal and cellular requirements for Fms signaling during zebrafish adult pigment pattern development. Development 2003, 130:817-833.

40. Albertson RC, Streelman JT, Kocher TD, Yelick PC: Integration and evolution of the cichlid mandible: the molecular basis of alternate feeding strategies. P Natl Acad Sci Usa 2005, 102:16287-16292.
41. Hoegg S, Boore $J$, Kuehl JV, Meyer A: Comparative phylogenomic analyses of teleost fish Hox gene clusters: lessons from the cichlid fish Astatotilapia burtoni. BMC Genomics 2007, 8:317

42. Panganiban G, Rubenstein J: Developmental functions of the Distal-less/Dlx homeobox genes. Development 2002, 129:4371-4386.

43. Ellies DL, Stock DW, Hatch G, Giroux G, Weiss KM, Ekker M: Relationship between the genomic organization and the overlapping embryonic expression patterns of the zebrafish dlx genes. Genomics 1997, 45:580-590.

44. Borday-Birraux V, Van der Heyden C, Debiais-Thibaud M, Verreijdt L, Stock DW Huysseune A, Sire J-Y: Expression of Dlx genes during the development of the zebrafish pharyngeal dentition: evolutionary implications. Evol Dev 2006, 8:130-141.

45. Debiais-Thibaud M, Germon I, Laurenti P, Casane D, Borday-Birraux V: Low divergence in $D / x$ gene expression between dentitions of the medaka (Oryzias latipes) versus high level of expression shuffling in osteichtyans. Evol Dev 2008, 10:464-476.

46. Beldade P, Brakefield PM, Long AD: Contribution of Distal-less to quantitative variation in butterfly eyespots. Nature 2002, 415:315-318.

47. Reed RD, Serfas MS: Butterfly wing pattern evolution is associated with changes in a Notch/Distal-less temporal pattern formation process. Curr Biol 2004, 14:1159-1166.

48. Brakefield PM, Gates J, Keys D, Kesbeke F, Wijngaarden PJ, Monteiro A, French V, Carroll SB: Development, plasticity and evolution of butterfly eyespot patterns. Nature 1996, 384:236-242

49. Sunkel CE, Whittle J: Brista: A gene involved in the specification and differentiation of distal cephalic and thoracic structures in Drosophila melanogaster. Roux's Arch Dev Biol 1987, 196:124-132.

50. Dong P, Chu J, Panganiban G: Coexpression of the homeobox genes Distal-less and homothorax determines Drosophila antennal identity. Development 2000, 127:209-216.

51. Gordon CT, Brinas I, Rodda FA, Bendall AJ, Farlie PG: Role of Dlx genes in craniofacial morphogenesis: D/x2 influences skeletal patterning by inducing ectomesenchymal aggregation in ovo. Evol Dev 2010, 12:459-473.

52. Stock DW, Ellies DL, Zhao ZY, Ekker M, Ruddle FH, Weiss KM: The evolution of the vertebrate Dlx gene family. P Natl Acad Sci Usa 1996, 93:10858-10863.

53. Terai $Y$, Morikawa N, Okada N: The evolution of the pro-domain of bone morphogenetic protein 4 (Bmp4) in an explosively speciated lineage of East African cichlid fishes. Mol Biol Evol 2002, 19:1628-1632.

54. Notredame C, Higgins DG, Heringa J: T-Coffee: a novel method for fast and accurate multiple sequence alignment. J Mol Biol 2000, 302:205-217.

55. Poirot O, O'Toole E: Tcoffee@igs: a web server for computing, evaluating and combining multiple sequence alignments. Nucleic Acids Res 2003, 31:3503-3506.

56. Swofford DL: Phylogenetic Analysis Using Parsimony (*and Other Methods). Sunderland: Sinauer Associates; 2003.

57. Geneious version 5.6 created by Biomatters. Available from http://www. geneious.com/.

58. Bruford MW, Hanotte O, Brookfield J, Burke T: Multilocus and single-locus DNA fingerprinting. In Molecular genetic analysis of populations: a practical approach. Edited by Hoelzel AR. Oxford: Oxford University Press; 1998:287-336.

59. Huelsenbeck JP, Ronquist F: MRBAYES: Bayesian inference of phylogeny. Bioinformatics 2001, 17:754-755.

60. Ronquist F, Huelsenbeck JP: MrBayes 3: Bayesian phylogenetic inference under mixed models. Bioinformatics 2003, 19:1572-1574.

61. Guindon S, Gascuel O: A simple, fast, and accurate algorithm to estimate large phylogenies by maximum-likelihood. Syst Biol 2003, 52:696-704.

62. Posada D: jModelTest: phylogenetic model averaging. Mol Biol Evol 2008 , 25:1253-1256.

63. Salzburger W, Meyer A, Baric S, Verheyen E, Sturmbauer C: Phylogeny of the Lake Tanganyika cichlid species flock and its relationship to the Central and East African haplochromine cichlid fish faunas. Systematic Biology 2002, 51:113-135.

64. Yang Z: PAML, A program package for phylogenetic analysis by maximum likelihood. Comput Appl Biosci 1997, 13:555-556.

65. Yang Z: PAML 4: phylogenetic analysis by maximum likelihood. $\mathrm{Mol}$ Biol Evol 2007, 24:1586-1591.

66. Nielsen R, Yang Z: Likelihood models for detecting positively selected amino acid sites and applications to the HIV-1 envelope gene. Genetics 1998, 148:929-936. 
67. Yang Z, Nielsen R, Goldman N: Codon- substitution models for heterogeneous selection pressure at amino acid sites. Genetics 2000, 155:431-449.

68. Yang Z, Wong W, Nielsen R: Bayes empirical Bayes inference of amino acid sites under positive selection. Mol Biol Evol 2005, 22:1107-1118.

69. Kosakovsky Pond SL, Frost S, Muse SV: HyPhy: hypothesis testing using phylogenies. Bioinformatics 2005, 22:676-679.

70. Massingham T, Goldman N: Detecting amino acid sites under positive selection and purifying selection. Genetics 2005, 169:1753-1762.

71. Ng P, Henikoff ST: SIFT: predicting amino acid changes that affect protein functions. Nucleic Acids Research 2003, 31:3812-3814.

72. Star B, Nederbragt AJ, Jentoft S, Grimholt U, Malmstrøm M, Gregers TF, Rounge TB, Paulsen J, Solbakken MH, Sharma A, Wetten OF, Lanzén A Winer R, Knight J, Vogel J-H, Aken B, Andersen $\varnothing$, Lagesen K, Tooming-Klunderud A, Edvardsen RB, Tina KG, Espelund M, Nepal C, Previti C, Karlsen BO, Moum T, Skage M, Berg PR, Gjøen T, Kuhl H, et al: The genome sequence of Atlantic cod reveals a unique immune system. Nature 2011, 477:207-210.

73. Sumiyama K, Irvine SQ, Stock DW, Weiss KM, Kawasaki K, Shimizu N, Shashikant CS, Miller W, Ruddle FH: Genomic structure and functional control of the D/x3-7 bigene cluster. P Natl Acad Sci Usa 2002, 99:780-785.

74. Force A, Lynch M, Pickett FB, Amores A, Yan YL, Postlethwait J: Preservation of duplicate genes by complementary, degenerative mutations. Genetics 1999, 151:1531-1545.

75. Sidow A: Gen(om)e duplications in the evolution of early vertebrates. Curr Opin Genet Dev 1996, 6:715-722.

76. Ohta T: Simulating evolution by gene duplication. Genetics 1987, 115:207-213.

77. Marotta M, Piontkivska $\mathrm{H}$, Tanaka H: Molecular trajectories leading to the alternative fates of duplicate genes. PLOS ONE 2012, 7:e38958.

78. Conrad B, Antonarakis SE: Gene duplication: a drive for phenotypic diversity and cause of human disease. Annu Rev Genom Human Genet 2007, 8:17-35.

79. Prince VE, Pickett FB: Splitting pairs: the diverging fates of duplicated genes. Nature Reviews Genetics 2002, 3:827-837.

80. Braasch I, Volff JN, Schartl M: The endothelin system: evolution of vertebrate-specific ligand-receptor interactions by three rounds of genome duplication. Mol Biol Evol 2009, 26:783-799.

81. Zhang J, Zhang Y-P, Rosenberg HF: Adaptive evolution of a duplicated pancreatic ribonuclease gene in a leaf-eating monkey. Nat Genet 2002 30:411-415.

82. Zhang J: Parallel adaptive origins of digestive RNases in Asian and African leaf monkeys. Nat Genet 2006, 38:819-823.

83. Dermitzakis ET, Clark AG: Differential selection after duplication in mammalian developmental genes. Mol Biol Evol 2001, 18:557-562.

84. Terai Y, Morikawa N, Kawakami K, Okada N: Accelerated evolution of the surface amino acids in the WD-repeat domain encoded by the hagoromo gene in an explosively speciated lineage of east African cichlid fishes. Mol Biol Evol 2002, 19:574-578.

85. Wagner GP, Lynch VJ: Evolutionary novelties. Current Biology 2010, 20:R48-R52

86. Prud'homme B, Gompel N, Carroll SB: Emerging principles of regulatory evolution. P Natl Acad Sci Usa 2007, 104:8605-8612.

doi:10.1186/1471-2148-13-153

Cite this article as: Diepeveen et al:: Sequence analyses of the distal-less homeobox gene family in East African cichlid fishes reveal signatures of positive selection. BMC Evolutionary Biology 2013 13:153.

\section{Submit your next manuscript to BioMed Central and take full advantage of:}

- Convenient online submission

- Thorough peer review

- No space constraints or color figure charges

- Immediate publication on acceptance

- Inclusion in PubMed, CAS, Scopus and Google Scholar

- Research which is freely available for redistribution

Submit your manuscript at www.biomedcentral.com/submit
Biomed Central 